\title{
INTERLAYER EXCHANGE COUPLING AND GIANT MAGNETORESISTANCE IN MAGNETIC MULTILAYERS
}

\author{
P.J.H. BLOEMEN \\ Department of Physics, Eindhoven University of Technology (EUT) \\ 5600 MB Eindhoven, The Netherlands
}

\begin{abstract}
An introduction is given on the subject of magnetic metallic multilayers. These artificial structures appear to display several fascinating physical phenomonena which are of fundamental as well as technological interest. Two examples, the magnetic coupling between ferromagnetic layers across a non-magnetic spacer layer and the giant magnetoresistance are emphasized in this article. For each of these, key experimental observations are reviewed followed by a discussion of the physical principles underlying the phenomenon.
\end{abstract}

PACS numbers: 75.30.Et, 75.50.Rr

\section{Introduction}

Magnetism, although a very old field of research, is still an extremely active area, which has come to play a very important role in modern life. One of the most illustrative examples is the ability of magnetic materials to store information through (controllable) changes in magnetization directions. This property is at the heart of today's computer technology (rigid disks, flexible disks), consumer electronics (tapes for audio and video apparatuses) and many other important applications (such as magnetic strips for personal data on credit cards, etc.). The performance of magnetic devices is obviously largely determined by the properties of the magnetic solid involved. Since these properties depend heavily on the type of atoms and their environment, a high degree of control of composition and structure is desired. It is therefore not surprising that most research being performed today is on man-made magnetic materials, for which this control is implicitly possible. An example of such artificially-prepared magnetic materials is formed by magnetic multilayers.

Multilayers (in their most simple form) consist of two materials arranged alternately in layers of certain thicknesses. They are mostly prepared by sputtering or molecular-beam epitaxy under ultra-high vacuum conditions. The progress in developing and refining these preparation-techniques in the last decade has been such that it is now possible to grow layers of very high purity and with extraordinarily good thickness-control (down to the monoatomic layer regime), for many 
material combinations. The fact that artificial multilayers thus prepared do not occur in nature suggests that, given the unique manipulation opportunities and unique geometry, they may be engineered (or tailored) to have properties which might not otherwise be attainable.

Several interesting and sometimes spectacular physical phenomena have been reported. Among these are dimensionality effects already exhibited by single films (i.e. multilayers are not necessary). An example concerns the temperature dependence of the magnetization, which can be affected considerably due to the reduction in the number of magnetic neighbours. Curie temperatures which are strongly reduced with respect to the bulk metals, with reductions of an order of magnitude for monolayers, have also been reported - see e.g. [1].

A second group of effects can be classified under proximity or interface effects, i.e. effects which occur due to the presence of a boundary between two different materials. One of the most striking phenomena in this category is the occurrence of an easy direction for the magnetization perpendicular to the plane of the magnetic layer. This discovery was particularly amazing, since magnetic films were always expected to have their magnetization oriented parallel to the film plane as a result of the strong demagnetizing fields created when tilting the magnetization out of the film plane. The phenomenon was quickly ascribed to an interface or surface anisotropy, which in fact had already been predicted in 1954 by Néel [2], who argued that atoms at the surface of a magnetic layer, due to their reduction in symmetry, should exhibit a considerably different magnetic anisotropy than their inner counterpart atoms.

A third group of effects concerns physical phenomena which only occur for systems consisting of at least two magnetic layers. One of these is the magnetic coupling between two ferromagnetic layers separated from each other by an intervening non-magnetic layer. Another fascinating and for application oriented researchers perhaps the most important effect, is the so-called giant magnetoresistance effect. It was observed that the electrical resistance of several multilayered systems decreased strongly upon applying a magnetic field.

There are these latter two phenomena which the present article is concerned with. Key experiments are reviewed including a discussion of the important physical principles which account for the observations. The text is introductory of character: no attempt has been made to be exhaustive. Those who are interested in a more broad discussion including several other interesting effects which are not adressed here such as exchange biasing, stabilization of crystal structures which are different from their equilibrium bulk-phase counterparts, mechanical effects, etc., are referred to [3-5] and to the two volumes of Ultrathin Magnetic Structures collecting a number of excellent reviews [6].

\section{Interlayer exchange coupling}

The topic of this section is the magnetic coupling which has been discovered to exist between ferromagnetic layers across non-magnetic interlayers.

The term magnetic coupling between magnetic moments (or spins) usually refers to an interaction energy which depends on the relative orientation of the magnetic moments. In the present case we are interested in the dependence of the 
energy $E$ on the relative orientation between macroscopic layer magnetizations. It appears that this situation can be described phenomenologically by the expression $E=-J \cos (\theta)$ with $\theta$ - the angle between the magnetization vectors of the interacting layers and $J$ - the strength of the interaction being positive for ferromagnetic coupling and negative for antiferromagnetic coupling. Note that this expression has the same functional form as the classical equivalent of the Heisenberg exchange interaction which acts on an atomic level. Higher order terms like the biquadratic exchange varying as $\cos ^{2}(\theta)$ may also occur but are commonly orders of magnitude smaller than the first order (bilinear) term except of course where this term becomes zero. In such situations effects of this term are actually observed experimentally, see e.g. [7]. However, it is beyond the scope of this paper to discuss the experiments and the models that focus on this term.

\subsection{Experimental observations, history}

The experimental studies of interaction phenomena in multilayer films date from at least 1902, according to Yelon [8]. An important problem in these studies is the practical difficulty in manufacturing ultrathin interlayer films without microscopic holes. The formation of such holes, through which the ferromagnetic layers can touch and form ferromagnetic bridges (so-called pinholes), is disastrous, since such structures will outweight any indirect antiferromagnetic coupling through their very strong direct ferromagnetic coupling. This is probably the reason why clear evidence for an indirect antiferromagnetic interlayer exchange coupling was not obtained until 1986 for the rare-earth transition metal multilayers Dy/Y [9], $\mathrm{Gd} / \mathrm{Y}$ [10], and for the $3 d$ transition metal system $\mathrm{Fe} / \mathrm{Cr} / \mathrm{Fe}$ [11]. Preceding this

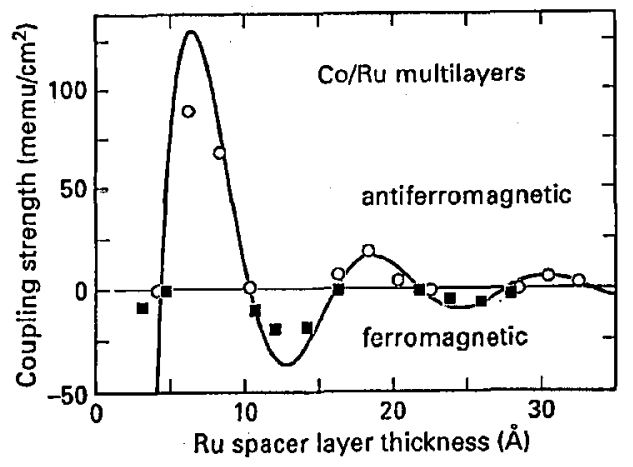

Fig. 1. The strength of the interlayer exchange coupling as a function of the Ru spacer thickness observed for sputtered $\mathrm{Co} / \mathrm{Ru}$ multilayers (after [14]).

study, the observed interlayer interactions were always found to be ferromagnetic and non-oscillatory (see e.g. [8] and for more recent studies [12]). The discovery of antiferromagnetic (AF) coupling in the $\mathrm{Fe} / \mathrm{Cr}$ system was followed in 1990 by the discovery of its oscillatory nature by Parkin et al. [13]. An example is shown in Fig. 1 displaying the behavior of the coupling strength as a function of the $\mathrm{Ru}$ spacer layer thickness for the Co/Ru system [14]. This behavior could be analyzed 
in terms of a single oscillation with a period $\Lambda$ of about $10 \AA$ and decaying with the Ru thickness $t$ as $1 / t^{2}$ :

$$
J=J_{0} \sin \left(\frac{2 \pi}{\Lambda} t+\phi\right) / t^{2} .
$$

Subsequently it was discovered that the phenomenon was a general feature: almost all non-magnetic $3 d, 4 d$ and $5 d$ transition metals were found to transmit an oscillatory interaction [15]. For many spacer materials about $10 \AA$ was found for the oscillation period. However, it soon appeared that this rather simple experimental situation which suggested a $10 \AA$ universal period was oversimplified. For $\mathrm{Cr}$ a long period of $18 \AA$ was found whereas for Os, which was not included in earlier experiments, also a significantly larger period of approximately $15 \AA$ was found [16]. Moreover, further experiments [17, 18], also inspired by theoretical predictions, showed that the oscillation periods depended on the specific growth orientation, i.e. on the orientation of the crystallographic planes with respect to the film normal, so that, even for a given element, no unique period could be defined. The situation became even more complicated after more refined experiments on samples having a considerably improved crystalline quality. From these it appeared that in addition to the above-mentioned long period oscillations also oscillations were present with very short periods down to 2 monolayers (MLs) for Cr and Mn (100) spacers [19, 20], i.e. the exchange coupling displayed a so-called multiperiodic coupling behavior. Other examples of such a behavior was observed for systems with $\mathrm{Cu}, \mathrm{Ag}$ and $\mathrm{Au}(100)$ spacers [21-23]. Here, the $\mathrm{Fe} / \mathrm{Au}(100)$ case, shown in Fig. 2, demonstrates this behavior most clearly. Three sets of two peaks are observed which can be analyzed as being a result of a superposition of long and a short period oscillation

$$
J(t) \sim\left(1 / t^{2}\right)\left[\sin \left(\frac{2 \pi t}{\Lambda_{1}}+\phi_{1}\right)+r \sin \left(\frac{2 \pi t}{\Lambda_{2}}+\phi_{2}\right)\right],
$$

the long period $\Lambda_{1}$ corresponding to the distance between the sets and the short period $\Lambda_{2}$ corresponding to the distance between the peaks within one set.

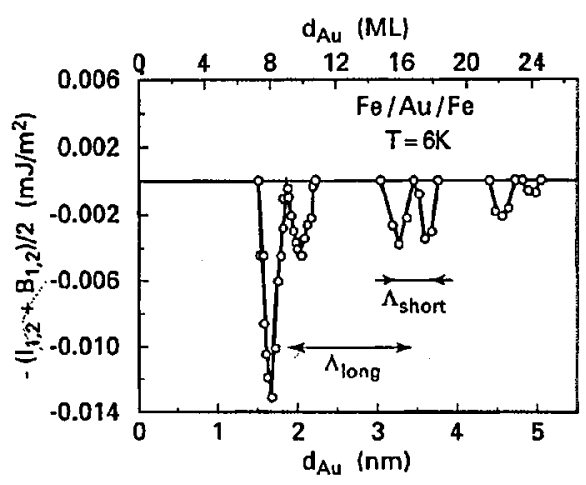

Fig. 2. Biperiodic oscillatory behavior of the interlayer exchange coupling for $\mathrm{Fe} / \mathrm{Au} / \mathrm{Fe}(100)$ trilayers (after [23]). 
For the present purpose of discussion the experiments mentioned so far are sufficient. Other important experimental observations will be addressed while discussing the physical origin of the oscillatory interlayer exchange interaction.

\subsection{Understanding the oscillatory coupling phenomenon}

In the physics of thin magnetic layers several coupling mechanisms are active. In this section we address the question which of them is relevant to our problem and how to explain the various experimental observations.

The most well-known example of a magnetic coupling which depends on the relative orientation between the magnetic moments is the long-range dipolar interaction. The interaction energy between two magnetic moments $m_{1}$ and $m_{2}$ is given by

$$
E_{\mathrm{dip}}=\frac{\mu_{0}}{4 \pi}\left[-\frac{m_{1} \cdot m_{2}}{r^{3}}+3 \frac{\left(m_{1} \cdot r\right)\left(m_{2} \cdot r\right)}{r^{5}}\right] .
$$

Here $r$ is the bonding vector of the two magnetic moments. Note that this interaction falls off monotonically as $1 / r^{3}$ with increasing distance $r$ between the dipoles.

In the case of atomic moments, a much more important interaction is the direct exchange interaction resulting from the overlap of the wave functions. Dependence on the overlap causes the interaction to fall off extremely rapidly with distance, and the interactivis is therefore often only considered for nearest-neighbor atoms. It originates physically from the Coulomb interaction combined with Pauli's principle, but can be written in terms of spin magnetic moments as

$$
E_{\text {exch }}=-2 J m_{1} \cdot m_{2} /\left|m_{1}\right|\left|m_{2}\right| .
$$

Here, $J$ denotes the strength of the interaction, which is positive for preferential parallel alignment (ferromagnetic) and negative for preferential antiparallel alignment (antiferromagnetic).

A third example known to occur in metallic systems are indirect exchange interactions. These are typically two orders of magnitude smaller than direct exchange interactions, and therefore usually manifest themselves only in cases where the latter are absent. This is the case in, for example, the rare-earth transition metals, where the $4 f$ wave functions carrying the magnetic moment are localized such that there is no overlap between them. The ordering in these systems is now governed by the indirect exchange interactions, leading to ordinary ferromagnets like Gd, but also to exotic helical spin structures such as in Dy. Another example is formed by dilute magnetic alloys such as $\mathrm{CuMn}$, where, due to the low $\mathrm{Mn}$ content, the distances between the Mn atoms are too large to allow overlap, and a peculiar spin-glass behavior can occur.

An important indirect exchange interaction which can account for the various observations, such as the helical structures and the spin-glass behavior, is the so-called RKKY interaction, named after Ruderman, Kittel, Kasuya and Yosida [24-26], who pioneered early theoretical work in this field. The remarkable but essential feature of this interaction is its oscillatory behavior. Contrary to, for example, the dipolar interactions, where two magnetic moments always 
couple with the same polarity* irrespective of the distance between them, the RKKY interaction is alternatingly ferromagnetic and antiferromagnetic with increasing distance. It appears that the interaction again can be written in the form of Eq. (4) [25], in which $J$ at large distances takes the form [26]:

$$
J \sim \cos \left(2 k_{\mathrm{F}} r\right) / r^{3} .
$$

It is clear that in order to explain the experimental observations the RKKY mechanism is the best candidate. The direct exchange interaction has a range which is too short whereas the dipolar interaction is non-oscillatory and independent of the spacer material.

To understand the mechanism of the RKKY interaction and how it works out in the layered geometry it is very instructive to consider the physics of a single interface between two non-magnetic metals. In Fig. 3 the situation is depicted schematically. Here, the horizontal coordinate represents the position normal to the interface whereas the vertical coordinate denotes the energy. If two metallic layers are brought into contact with each other their Fermi-levels will line up. Depending on the difference of the initial Fermi-levels of the two metals the energy bands will have a certain relative position with respect to each other. In the free-electron approximation the resulting change in electronic structure at the interface can be described by a single potential step, as shown in Fig. 3. From here on we will continue to treat the metals as if they are completely free-electron like. Of course, this does not correspond to reality but it makes the discussion very transparent. Moreover, the most important consequences for the features of the coupling phenomenon are not lost by this approximation.

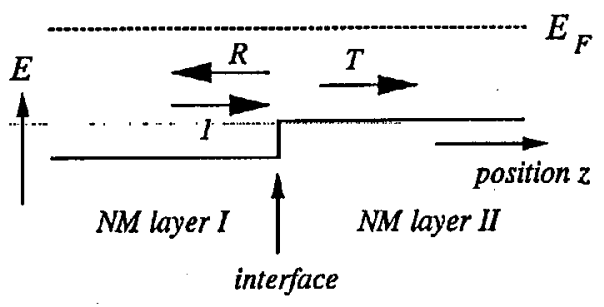

Fig. 3. Potential energy as a function of position perpendicular to an NM/NM interface. The broken line indicates the position of the Fermi level which is typically $7 \mathrm{eV}$ above the bottom of the conduction band (the solid line).

Within the free-electron approximation the situation in Fig. 3 may be recognized as a standard quantum-mechanics text book problem. The behavior of electrons may be characterized by ordinary plane waves of the form $\exp \left(\mathrm{i} k_{x} x+\right.$ $\left.\mathrm{i} k_{y} y+\mathrm{i} k_{z} z\right) \equiv \exp \left(\mathrm{i} k_{\|} r_{\|}\right) \exp \left(k_{z} z\right)$ in which $k_{x}, k_{y}$, and $k_{z}$ represent the wave vectors parallel to the interface (the $x, y$-directions) and perpendicular to the interface (the $z$-direction). Here, $k_{\|} \equiv\left(k_{x}, k_{y}\right)$ and $r_{\|} \equiv(x, y)$. The general solution

${ }^{*}$ Antiferromagnetically or ferromagnetically, depending on the direction of the moments relative to the bonding axis. 
of the Schrödinger wave equation in the region of layer I can be written as the sum of an incident and a reflected wave

$$
\psi_{k}=\left[\exp \left(\mathrm{i} k_{z} z+R \exp \left(-\mathrm{i} k_{z} z\right)\right] \exp \left(\mathrm{i} k_{\|} r_{\|}\right)\right.
$$

with $R$ - the complex reflection coefficient. We may now directly infer the effect of the potential step on the electron density $\rho$ in layer I. Since in a metallic solid the electrons occupy a range of energies or wave vectors ranging from 0 up to the Fermi wave vector $k_{F}$ we have to sum over all these electrons to obtain the net effect

$$
\rho(z)=\frac{1}{4 \pi^{3}} \int_{0}^{k_{\mathrm{F}}}\left|\psi_{k}\right|^{2} \mathrm{~d}^{3} k .
$$

Substitution of Eq. (6) into Eq. (7) immediately yields that the oscillatory contribution to $\rho$ is given by

$$
\rho(z)=\frac{R}{4 \pi^{2}} 2 k_{\mathrm{F}} \cos \left(2 k_{\mathrm{F}} z\right) / z^{2}
$$

with $z$ - the distance from the interface and $R$ - the reflection coefficient at the Fermi energy. We see that the presence of a potential step induces an oscillation in the electron density. This is a general feature of metals: any discontinuity in the potential induces such an oscillation. For impurities for example this will have a spherical form like the one in Eq. (5). In the case of an interface between a non-magnetic metal and a ferromagnetic metal one has to distinguish the electrons according to their spin direction. In a ferromagnet they are not equivalent because the bands are shifted relative to each other, see Fig. 4. The result is that the step-height at the interface will be different for spin-up and spin-down electrons. This has the consequence that the electron densities of the spin-up and spin-down electrons do not cancel anymore as they did for a non-magnetic/non-magnetic interface but that near the interface apart from an electron-density oscillation also an oscillation in the spin-density $\sigma(z)=\rho \downarrow-\rho \uparrow$ occurs having the same functional dependence as $\rho$ in Eq. (8). To make the final step towards interlayer coupling one may imagine a probe spin or magnetic moment moving away from the interface. As a function of distance this spin will "experience" a net magnetic moment induced locally in the sea of electrons whose sign changes from being parallel to the magnetization of the ferromagnet (locally more spin-up electrons) to being antiparallel to the magnetization of the ferromagnet (locally more spin-down electrons). Consequently the probe magnetic moment will (due to its contact exchange interaction with the polarized electrons) preferably align its magnetic moment parallel or antiparallel to the ferromagnet magnetization. As a next ștep one may replace the probe spin by a ferromagnetic layer yielding an interlayer exchange coupling which oscillates. Of course, in this case the second ferromagnetic layer also induces a spin-density oscillation which will interfere with the first one. A theoretical model by $\mathrm{P}$. Bruno took this effect into account. By considering the energy associated with the in general multiple reflections which electron waves may undergo in the spacer he was able to make quantitative predictions for the interlayer exchange coupling strength [27]. It turns out that the square of the reflection coefficient $R^{2}$ determines the strength of the coupling, to be more precise $(\Delta R)^{2}$ with $\Delta R$ the 


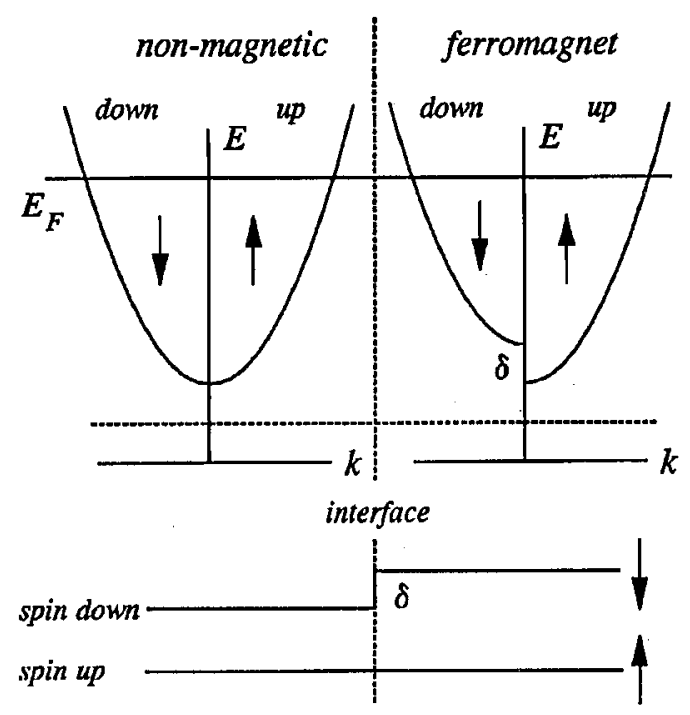

Fig. 4. Situation of a NM/FM interface. The potential energy as a function of position as well as the parabolic dispersion relations are shown for both spin-directions.

difference in reflection coefficients for the two spin-directions. This will have important consequences for the strength of the coupling as we will discuss later. The other features remain unaltered. They can be inferred from Eq. (8): the period $\Lambda$ of the interlayer exchange coupling is determined by the length of the vector spanning the diameter of the Fermi-sphere: $\Lambda=2 \pi / 2 k_{\mathrm{F}}$; the decay occurs with the square of the distance or layer thickness: $1 / t^{2}$ like in Eq. (1). However, a few remarks need to be made.

Although the $1 / t^{2}$ decay seems to agree with several experiments this is not generally the case. Often discrepancies occur which are attributed to sample imperfections. It is thought that these result in diffusive electron scattering causing a loss of coherence of these electrons with the unscattered ones. It may be clear that the probability of scattering increases with spacer thickness yielding a more rapid fall off with coupling strength with $t$.

If one calculates the oscillation period $\Lambda=\pi / k_{\mathrm{F}}$ for metals like $\mathrm{Cu}$ one obtains relatively small values of typically $2 \AA$. This seems at first glance in contradiction with the rather long periods of about $10 \AA$ observed in many experiments (see the previous section). However, this problem has been resolved by realizing that in a coupling experiment the spacer layer thickness cannot be varied continuously but only in discrete steps of one atomic monolayer. This is illustrated in Fig. 5 [28]. Here, a rapid oscillation (with period $\lambda=\pi / k_{\mathrm{F}}$ ) is shown (full curve). The sampling or measuring at the discrete lattice planes (the solid points) causes the rapid oscillation to emerge as an oscillation with a longer period of indeed near the observed $10 \AA$ [28-30]. A last remark concerns the multiplicity of the oscillations. From the above it may appear that merely one oscillation is possible. Clearly this is at variance with the experiments. Figure 2 for example shows that 


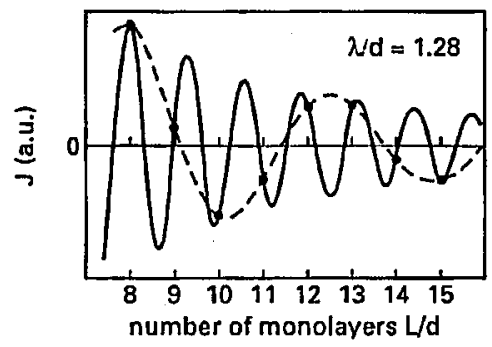

Fig. 5. Coupling $J(L)$ as a function of the interlayer thickness expressed in units of interplanar spacing $d$. Full curve: rapid oscillation with period $\lambda=1.28 d$; broken curve: measured oscillatory coupling with a period $\Lambda=\lambda / 0.28 \approx 3.57 \lambda$ (after [28]).

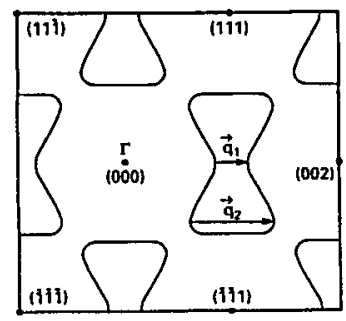

Fig. 6. Cross-section of the $\mathrm{Cu}$ Fermi surface relevant for magnetic coupling across $\mathrm{Cu}$ interlayers grown in the $(100)$ orientation. The "dogbone"-shaped hole in the FS exhibits two extremal parts i.e. the coupling may be a superposition of two oscillations. The oscillation periods are determined by the length of the two $q$ vectors, spanning these extremal parts.

the exchange coupling can be a superposition of two oscillations. This difficulty has been resolved by among others P. Bruno who showed that apart from the vector spanning the Fermi sphere also the vector connecting the spheres in the repeated zone scheme gives rise to an oscillation [31]. Furthermore, it was shown that in general for arbitrary Fermi surfaces of non-free electron-like metals in principle each vector (parallel to the growth direction) which spans extremal parts of the Fermi surface (FS) may give rise to an oscillation [31,32]. Figure 6 illustrates this for the relevant cross-section of the $\mathrm{Cu}$ FS for (100) growth. Here, two extremal vectors can be identified with their lengths $q_{i}$ related to the period $\Lambda_{i}$ by $q_{i}=2 \pi / \Lambda_{i}$. Indeed, for the $\mathrm{Co} / \mathrm{Cu} / \mathrm{Co}(100)$ system a superposition of two oscillatory contributions was found like in the $\mathrm{Fe} / \mathrm{Au}(100)$ case of Fig. 2. We mention that the FS cross-section for $\mathrm{Au}(100)$ is similar to that shown in Fig. 6.

It may now also be clear that the periods depend on the growth orientation: FS cross-sections taken along different directions will differ from each other. Many experiments have been devoted to confirm the view that the periods are determined by the topology of the FS. Examples include experiments where the FS dimensions and topology of the spacer material was varied by studying spacers of different material ( $\mathrm{Cu}, \mathrm{Ag}, \mathrm{Au}$ [21-23]), having a different growth orientation 
$(\mathrm{Cu}(100,111,110)[21,17,18])$, having a different crystal structure (e.g. fcc versus bcc $\mathrm{Cu}$ [21]) or having a different band filling or electron density. The latter was achieved by adding a second element in the spacer, i.e. through alloying, see e.g. [33]. In general, all experiments support the FS picture so that at present the periods of the oscillatory coupling are reasonably well understood.

Currently, the research is directed towards understanding the strength and phase of the oscillatory coupling. Here, an important insight, viz. that the coupling phenomenon is not an interface effect but a property of the sandwich as a whole, was brought about by experiment $[34,35]$ as well as theory $[36,27,37]$. This conclusion directly follows from our discussion of the physics of a single interface. As we have seen and as has been proved by Bruno [27] the strength of the coupling is directly determined by the complex reflection coefficients at the ferromagnet/non-magnet (FM/NM) interface. This has several consequences. The first is that the coupling should oscillate also as a function of the thickness of the ferromagnetic layer. This is because the effective reflection coefficient of a layer of finite thickness depends on the interferences of electron waves reflecting from the front interface and from the back interface of the layer, see [37]. As mentioned, in experiments on the $\mathrm{Co} / \mathrm{Cu}(100)$ [34] and the $\mathrm{Fe} / \mathrm{Cr}(100)$ system [35] such oscillations were actually observed. A second consequence, being in principle an extension of the first one, is that the reflection coefficients or alternatively the coupling strength are not only modulated by interferences within the FM layers but in principle by interferences in every layer in the sample. Recently it has been shown that the coupling oscillates with the thickness of a non-magnetic layer capping the FM/spacer/FM trilayer structure [38]. Clearly, this experiment shatters the thought that the trilayer structure is supposed to form the active part dictating the coupling phenomenon fully. Another important consequence involved the insight that the presence or absence of energy band gaps for the FM at relevant parts in $k$-space at the Fermi level may drastically alter the reflection coefficients. It is clear that when a gap is present electrons cannot propagate into the FM layer since no states are available. Hence, they are reflected totally. Here, the phase of the reflection coefficient and thus the coupling is a sensitive function of the electron energy relative to the center of the gap. Recently, phase shift experiments, employing different FM compositions with consequently different positions of the energy gaps, were performed and provided strong support for this picture [39].

To conclude this section we remark that, although significant progress has been made in the understanding of the coupling phenomenon still a number of unresolved problems remain. For instance, multiperiodic behavior has not been detected so far for almost all transition metal spacers with unfilled $d$-bands (with the exception of $\mathrm{Cr}$ and $\mathrm{Mn}$ ); predicted intrinsic biquadratic coupling oscillations still require experimental confirmation; discrepancies between experiments and first principles band structure calculations regarding the strength; the lack of a model properly taking into account diffusive electron scattering effects; the lack of models taking into account the effect of a reduction of the ordering in the ferromagnet as a result of finite temperatures. Finally, we mention that convincing coupling experiments across semi-conducting or insulating spacers are still lacking. 


\section{Giant magnetoresistance}

The topic of this section is the giant magnetoresistance (GMR) effect: a drastic change in electrical resistivity when applying a magnetic field. This is a novel magneto-transport phenomenon which has no counterpart in nature: it is observed for samples consisting of ferromagnetic entities which are separated from each other by non-magnetic material in such a way that the relative magnetization directions of the entities can be changed by an applied magnetic field of moderate strength. The most important systems which show this behavior are magnetic multilayers consisting of a stack of alternating ferromagnetic and non-magnetic layers. The phenomenon also occurs in so-called granular systems in which ferromagnetic clusters are dissolved in a non-magnetic matrix. However, we restrict our attention to the multilayer systems since in these structures the phenomenon is most pronounced and more importantly because these systems allow more systematic investigations of the effect: the magnetization direction and size of each individual entity (ferromagnetic layer) as well as the distance between the entities can be controlled in a much higher degree than in the granular systems. Also from application point of view the layered structures are more interesting. Here the GMR effect is employed in magnetic field sensors in the read heads of data recording devices.

The first part of the section presents a selection of experimental observations which are important for the understanding of the phenomenon. The second part is devoted to a discussion of the underlying physical mechanism. Rather than giving mathematical treatments an attempt is made to keep the discussion as qualitative as possible. Because the subject is an ongoing activity in the community since about 1988 several review papers on this subject have already been written and are in preparation each emphasizing different aspects of the problem [40-43].

\subsection{Experimental observations}

The first indication for the novel magnetoresistance (MR) effect occurring in magnetic metallic multilayers was obtained in 1988 by Binasch et al. in $\mathrm{Fe} / \mathrm{Cr} / \mathrm{Fe}$ trilayers [44] and in the same year by Baibich et al. [45] who observed a very large effect but in this case for multilayers of $\mathrm{Fe}$ and $\mathrm{Cr}$. The typical behavior Baibich et al. observed is shown in Fig. 7. Here it is seen that the resistance (normalized to the zero field resistance) dramatically drops, in this case by a factor of about 2, upon application of a magnetic field. Because this novel effect was very large compared to the known MR effects the effect was labeled giant magnetoresistance. From accompanying studies of the magnetization as a function of the magnetic field (not shown here) it became clear that the resistance change correlated with the relative orientations of the magnetizations in the successive Fe layers. At zero field i.e. the high resistance state, the magnetizations are aligned antiparallel with respect to each other due to the existence of an antiferromagnetic coupling between the Fe layers across the $\mathrm{Cr}$ layers. Upon increasing the magnetic field this antiparallel state changes gradually through continuous rotation of the magnetizations towards the direction of the magnetic field until the saturated state is reached. Here, all Fe magnetizations are aligned parallel to the magnetic field and the state of low resistance is obtained. 


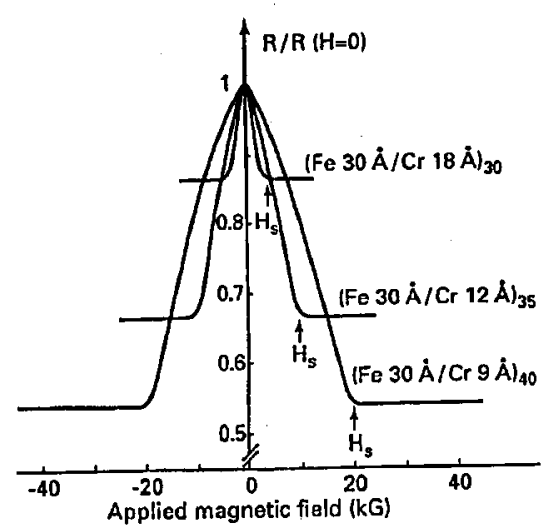

Fig. 7. Resistivity versus magnetic field for several antiferromagnetically coupled $\mathrm{Fe} / \mathrm{Cr}(100)$ multilayers. $H_{\mathrm{s}}$ is the field needed to saturate the magnetization (after [45]).

It is clear that the field at which the saturation occurs is a measure for the strength of the AF coupling and in fact this field, also indicated by $H_{\mathbf{s}}$ in Fig. 7, is often used for coupling strength determination.

Further research showed that the GMR effect was not restricted to the $\mathrm{Fe} / \mathrm{Cr}$ case but appeared to be a general phenomenon displayed by many combinations of the magnetic and non-magnetic transition metal elements [13]. For Co/Cu multilayers an example is shown in Fig. 8 [46] where it is seen that the MR ratio, defined as the relative change in resistance from zero field $R_{0}$ to high fields $R_{\text {sat }}$ : $\left(R_{0}-R_{\text {sat }}\right) / R_{\text {sat }}$ displays an oscillatory behavior with the $\mathrm{Cu}$ spacer thickness. Although this behavior mimics the behavior of the oscillatory interlayer exchange coupling exactly, it has become clear from several experiments that no obvious relation exists between antiferromagnetic coupling and giant magnetoresistance. The fact that the MR ratio goes to zero at for example a $\mathrm{Cu}$ spacer thickness of $13 \AA$ in the above example in Fig. 8 is merely a result of the fact that the polarity of the coupling is ferromagnetic at this thickness, preventing the occurrence of an antiparallel orientation between the magnetizations in the present experimental system. Speriosu et al. for example, proved experimentally, using clever spin-engineering experiments in which they were able to create antiparallel orientations also for spacer thicknesses where the coupling was ferromagnetic, that GMR occurs irrespective of the polarity and magnitude of the interlayer exchange coupling [47]. Therefore, at present it is accepted that GMR has no obvious relation to exchange coupling. A relation to quantum size effects which of course underlie the coupling phenomenon and which may also affect the resistance, is still an issue of debate where convincing experimental confirmation still lacks. To conclude this section we summarize a number of important features of the GMR phenomenon without discussing them individually.

The magnitude of the GMR is strongly material dependent. Values of $0.1 \%$ for $\mathrm{V}$ and Mo based multilayers are found whereas the record value today is $220 \%$ for the $\mathrm{Fe} / \mathrm{Cr}$ system [48]. Closer examination of the various multilayer systems which have been studied so far, learns that for the magnitude of the GMR effect 


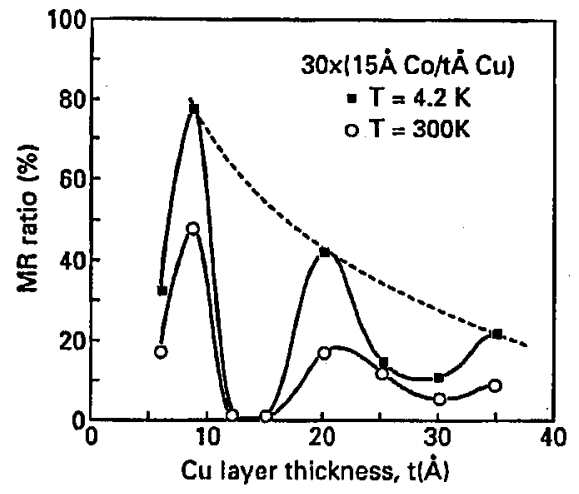

Fig. 8. Magnetoresistance ratio as a function of the $\mathrm{Cu}$ layer thickness observed for $\mathrm{Co} / \mathrm{Cu}$ multilayers (after [46]).

the material combination is crucial. For instance the use of $\mathrm{Cr}$ does not guarantee a high MR ratio. The $\mathrm{NiFe} / \mathrm{Cr}$ system, for example, exhibits rather small MR ratios of only $0.1 \%$ [49]. Also the choice of Fe as a ferromagnet does not guarantee a high $\mathrm{MR}$ ratio. Here, the Fe/Ag system forms a counter example, no appreciable MR was observed for this system [50]. Preparation details play a significant role. Many examples demonstrate that for the same multilayer system different values are obtained by different research groups using a different or even the same deposition technique [42]. This indicates that crystallinity and interface roughness may play a role. Also it has been found that the individual layer thicknesses, the use of baseor cap-layers, the number of multilayer repetitions and other compositional details influence the observed MR ratio. Finally, an important experimental observation is that the GMR effect decreases with increasing temperature. Again it is mentioned that this list is not exhaustive, but for the present scope of this article it is sufficient.

\subsection{Understanding the giant magnetoresistance phenomenon}

It is clear that for the GMR effect, being a particular electrical transport problem, electron scattering will be at the heart of the effect. In the paper of Baibich et al. [45] the hypothesis has been conjectured that the GMR effect originates in spin-dependent scattering of the conduction electrons i.e. there are different scattering probabilities and therefore different resistivities, $\rho \uparrow$ and $\rho \downarrow$ for the two spin directions - an interpretation that nowadays is widely accepted. With this hypothesis, the occurrence of an MR effect can be understood straightforwardly by considering the "trajectories" of the spin-up $\left(s_{z}=+\frac{1}{2}\right)$ and spin-down $\left(s_{z}=-\frac{1}{2}\right.$ ) electrons for the two relevant cases (i) the case where the magnetizations are aligned antiferromagnetically and (ii) for the saturated case with parallel alignment of the magnetizations. Figure 9 shows these two situations. In the parallel case, the spin-up electrons are weakly scattered in all layers and therefore. carry a large part of the electric current. In contrast the spin-down electrons are strongly scattered and therefore contribute little to the current. In the antiparallel case, an electron that initially is a spin-up electron for one magnetic layer will be a spin-down electron for the adjacent ferromagnetic layer since that layer has a 


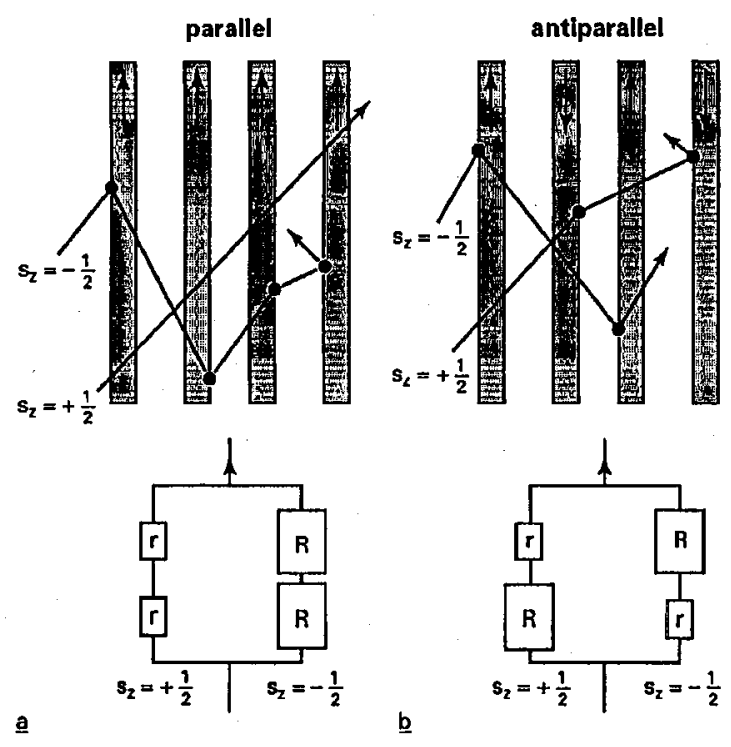

Fig. 9. Schematic picture to illucidate how spin-dependent scattering gives rise to a difference in conductivity between the cases that the layer magnetizations in a multilayer are ordered (a) ferromagnetically (the low resistance state) and (b) antiferromagnetically (the high resistance state). The upper figures represent the electron "trajectories" for both species of electrons $\left(S_{z} \pm \frac{1}{2}\right)$. The arrows denote the magnetization directions and a solid point represents a scattering event. The scattering probability is assumed to be lower for the majority electrons i.e. for electrons with their spin-direction parallel to the local magnetization. The lower figures represent the equivalent resistor networks (after [40]).

reversed magnetization axis and consequently a reversed quantization axis. The result is that electrons of both species are alternately strongly and weakly scattered as they cross the successive ferromagnetic layers. It is immediately clear that the saturated case corresponds to a lower resistance than the antiferromagnetic case because of the short circuit path which exists for one species of electrons in that case. Hence spin-dependent scattering is sufficient to explain the occurrence of a magnetoresistance effect when one causes the multilayer system to undergo a transition from an antiferromagnetic to a ferromagnetic state by application of a magnetic field. In addition the sign of the effect i.e. the resistance decreases with increasing field, is predicted correctly.

A first simple model describing the GMR in more quantitative terms can be obtained by application of the so-called two-current model - a simple resistor model which has been employed successfully in the past to describe the electrical conduction of dilute ternary ferromagnetic alloys [51]. In this model the conduction is described by dividing the current according to the spin-direction of the electrons in two independent spin-currents in parallel. A requirement necessary for this subdivision to be meaningful is the absence of mechanisms which result in spin-flip 
scattering which would mix the two currents. This requirement is usually fullfilled at low temperatures. An additional requirement for the multilayer case concerns the mean free paths of both electrons: they should be large compared to the layer thicknesses. With these assumptions the antiferromagnetic and ferromagnetic case can be modeled by the equivalent resistor networks shown in the bottom part of Fig. 9. The total resistivity $\rho_{\mathrm{F}}$ in the ferromagnetic case (with two different resistances in parallel) is then written as [40]:

$$
\rho_{F}=\rho \uparrow \rho \downarrow /(\rho \uparrow+\rho \downarrow),
$$

whereas for the antiferromagnetic case $\left(\rho_{\mathrm{AF}}\right)$, with both channels having the same conductivity the resistivity is given by [40]:

$$
\rho_{\mathrm{AF}}=(\rho \uparrow+\rho \downarrow) / 4 .
$$

The MR ratio $\Delta R / R$ defined as $\left(\rho_{\mathrm{AF}}-\rho_{\mathrm{F}}\right) / \rho_{\mathrm{F}}$ is then given by

$$
\frac{\Delta R}{R}=\frac{(1-\alpha)^{2}}{4 \alpha}
$$

with $\alpha \equiv \rho \downarrow / \rho \uparrow-$ a parameter representing the degree of spin-asymmetry. The qualitative understanding of the GMR effect has now been reduced to the question why $\rho \downarrow \neq \rho \uparrow$ i.e. to the original hypothesis of spin-dependent scattering. Obviously, this question draws on the microscopic origin of the GMR effect where e.g. the dependence on the type of materials is hidden in the $\alpha$ parameter. However, we remark that one should not draw the conclusion that the magnitude of the GMR effect can be described by this single $\alpha$ parameter. This deceptively simple looking result is caused by several over-simplifications in the two-current model as there are the assumption of very large mean free paths and the fact that for example interface and bulk scattering are not distinguished. Before we address the question regarding the origin of the spin-dependent scattering we illucidate this point by considering the consequence of relaxing, for example, the assumption on mean free paths. The spacer thickness dependence of the GMR demonstrates this most clearly.

Figure 10 shows the typical behavior for the MR. It is clear that in practice the GMR effect will go to zero for large spacer thicknesses since on the one hand scattering in the spacer reduces the flow of electrons from one ferromagnetic layer to the other: an electron should sample at least two ferromagnetic layers and on the other hand with increasing spacer thickness an increasing portion of the current will shunt through the spacer without passing through any of the ferromagnetic layers. From this example it already emerges that the real situation is considerably more complex than suggested by the two-current model (Eq. (11)). In practice the GMR is determined by a complex interplay of several factors including the different conductivities or resistances of the layers composing the multilayer, the inhomogeneous distribution of scattering centers across the sample due to the presence of boundaries and interfaces, each with a possibly different roughness, the several other possible types of microstructure related defects, the accompanying scattering processes such as diffusive scattering, specular reflective scattering at interfaces or transmission through interfaces, all with a certain probability of occurrence which in general depends on the spin-direction and on the angle of incidence relative 


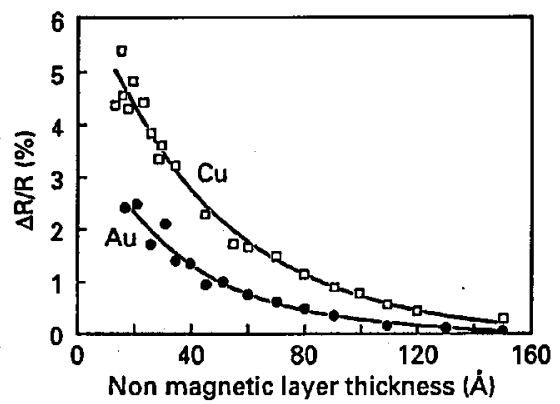

Fig. 10. Magnetoresistance versus thickness of the non-magnetic spacer layer for $\mathrm{Au}$ and $\mathrm{Cu}$ spacer materials (after [52]).

to the normal of the layers and which may conserve or flip the spin-direction. All these factors culminate in a certain current density which varies as a function position along the growth direction across the multilayer stack thereby causing certain parts of sample to contribute more effectively to the GMR effect than other parts.

In literature a multitude of models are proposed varying from semi-classical to fully quantum-mechanical, with adjustable parameters or from first principles, each having its own formalism and emphasizing or neglecting the several aspects to a different degree. It is far beyond the scope of this article to discuss these models and their successes and shortcomings here. Rather than doing this we refer to the review papers which we already mentioned and focus in the remaining part of the paper on the microscopic origin of the GMR effect i.e. the mechanisms that may underlie the spin-dependent scattering $(\rho \downarrow \neq \rho \uparrow)$. An important question in this respect is, for example, which materials properties account for a large spin-asymmetry in the conduction or scattering or alternatively can materials combinations be predicted which display a high GMR effect.

It is well known that for electrical transport problems the electronic structure of the material plays a crucial role. Ferromagnetic metals like $\mathrm{Fe}$, Co and Ni differ in this respect from ordinary metals such as $\mathrm{Al}$ and $\mathrm{Cu}$ for two reasons: $\mathrm{Fe}, \mathrm{Co}$ and $\mathrm{Ni}$ are transition metals having unfilled $d$-bands and they are ferromagnetic: one needs to distinguish the electrons according to their spin-direction: i.e. the two spin-bands are shifted along the energy axis relative to each other. In Fig. 11 a schematic representation of the conduction band density of states $N(\epsilon)$ as a function of energy $\epsilon$ is shown. The shift results in an unequal filling of the $d$-bands which in turn leads to a non-zero magnetization which is of course at the origin of ferromagnetism of these metals. Contrary to the magnetism (carried by the $d$-electrons), the electrical transport in these metals is primarily carried by the electrons in the $s p$-bands because they are broad, having a low effective mass (the $d$-bands are narrow with a high effective mass). Although the $s p$-bands in themselves do not exhibit a spin-asymmetry ${ }^{\dagger}$, the degree of conductivity does

\footnotetext{
TThe slight magnetic polarization which may be induced in the bands by exchange interaction with the $d$-electrons is of little importance here since the density of states for the $s p$-electrons is relatively small.
} 


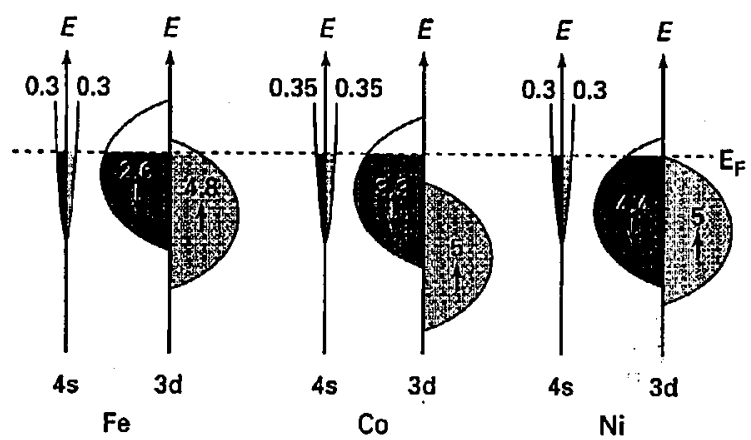

Fig. 11. Schematic diagram of the densities of states versus energy for the $s$ and $d$-bands of the transition metal ferromagnets $\mathrm{Fe}, \mathrm{Co}$ and $\mathrm{Ni}$. The number of electrons in the spin-down (left) and spin-up (right) bands are also shown (after [53]).

depend on the spin-direction. This is because the probability of scattering for these electrons is proportional to the density of states for the final states which in turn is enhanced relative to the noble metals because of the presence of $d$-states at the Fermi level and, more importantly is spin-dependent (the Mott mechanism [54]). Of course, the enhancement explains why the resistivity of transition metals like $\mathrm{Pd}, \mathrm{Pt}, \mathrm{Ru}$ etc. is so high and why $\mathrm{Cu}, \mathrm{Ag}$ and $\mathrm{Au}$ are such good conductors: their $d$-bands are completely filled and only $s-s$ scattering is possible with a much smaller probability: $N_{\mathrm{s}}\left(\epsilon_{\mathrm{F}}\right) \ll N_{d}\left(\epsilon_{\mathrm{F}}\right)$. Clearly, the spin-asymmetry of the $d$-bands in the case of $\mathrm{Fe}$, Co and $\mathrm{Ni}$ is important to the GMR effect.

Within the free-electron approximation, the above can also be expressed in terms of the well-known Drude formula

$$
\rho_{\sigma}=m_{\sigma} / n_{\sigma} e^{2} \tau_{\sigma}
$$

Here, $\rho_{\sigma}, m_{\sigma}, n_{\sigma}$, and $\tau_{\sigma}$ are the resistivity, effective mass, electron density and relaxation time, respectively separated for the electrons into the two spin-bands $\sigma$. The relaxation time, being inversely proportional to scattering probability, is related to the spin-dependent density of states $N_{\sigma}\left(\epsilon_{\mathrm{F}}\right)$ at the Fermi energy $\epsilon_{\mathrm{F}}$ by [55]:

$$
\tau_{\sigma}^{-1} \sim\left|V_{\sigma}\right|^{2} N_{\sigma}\left(\epsilon_{\mathbf{F}}\right)
$$

with $\left|V_{\sigma}\right|$ the matrix element of the perturbing potential connecting the initial and final states. In the matrix element the important physical information about the scattering processes such as scattering at phonons, vacancies, interstitials, dislocations and other possible defects, is contained. Although the scattering process itself and consequently the matrix element may be spin-independent, as in the above examples, Eq. (13) expresses that the corresponding relaxation times or mean free path lengths will still be spin-dependent and thus contribute to magnetoresistance. However, though it is still an issue of debate, it has been argued that for GMR the above-mentioned structural (spin-independent) randomness, having its primary source in the bulk of the layers, may be of less importance. It is thought that the dominant role is played by the spin-dependent randomness occurring at the ferromagnet/non-magnet interfaces because of roughness or interdiffusion. In this case the perturbation potentials set up by for example a step at the FM/NM 
interface or a magnetic impurity atom diffused into the non-magnetic layer, will evidently be spin-dependent. It is this additional spin-dependence (superimposed on the spin-asymmetry in the density of states) which forms the basis for the supposition that this interface randomness dictates the GMR.

From the foregoing it may be clear that the latter potentials will depend on the electronic structure and on the magnetism of the atoms involving the perturbation. Formulated in these terms a requirement for a large GMR effect is that for one spin-direction the perturbation in the electronic properties is negligible (the microscopic equivalent of the earlier-mentioned short circuit) whereas for the other spin-direction a maximum difference should be present. The fact that it is the perturbation or alternatively difference that is of importance expresses that indeed, as the experiments suggested, the material combination is crucial and not the individual elements. In order to predict which material combinations will yield large GMR ratios one could compare the electronic structures of the bulk metals. For the $\mathrm{Fe} / \mathrm{Cr}$ case the corresponding densities of states are compared in Fig. 12.

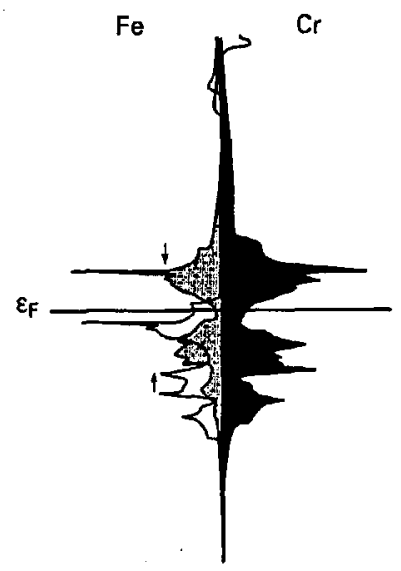

Fig. 12. Calculated densities of states for $\mathrm{Fe}$ and $\mathrm{Cr}$ with their respective Fermi energies aligned. Note that the Fe spin-up density of states, the shaded portion on the left, matches the $\mathrm{Cr}$ spin-up density of states, the shaded portion on the right divided by two (after [56]).

Here, it is seen that for the spin-up electrons the density of states of the $\mathrm{Fe}$ and of the $\mathrm{Cr}$ match very well whereas for the spin-down electrons this match is far from perfect. One could also consider the asymmetry in the subband resistivities $\rho \downarrow$ and $\rho \uparrow$ as determined from the residual resistivities measured for binary alloys of for example in our case FeCr. For the present example the relatively low value for $\rho \uparrow$ and the significant value for $\rho \downarrow$ is again favorable for a large GMR [51]. Therefore for $\mathrm{Fe} / \mathrm{Cr}$, being todays record GMR system [48], this simple procedure works out quite well. However, this appears to be fortuitous and not generally applicable. The $\mathrm{Fe} / \mathrm{V}$ multilayer system forms in this case the counter example since for this combination both arguments apply also whereas in contrast to $\mathrm{Fe} / \mathrm{Cr}$ the observed 
MR values are relatively low. Recently a significant step towards understanding this problem has been made [57, 58]. Here, band-structure calculations [58] for rough interfaces indicated that the error in the intuitive reasoning originates in the fact that the magnetism and electronic properties of $\mathrm{V}$ atoms either in the bulk or completely dissolved in an Fe matrix are significantly different and not representative for $\mathrm{V}$ atoms located at a (rough) $\mathrm{Fe} / \mathrm{V}$ interface. Furthermore, the insight in the microscopic picture of spin-dependent scattering was further increased by establishing a relationship between the spin-dependent scattering potential $V_{\sigma}$ and the interface magnetism in terms of two relatively simple quantities: the atomic number difference $\left(Z_{A}-Z_{B}\right)$ and the magnetic moment difference $\left(m_{A}-m_{B}\right)$ between the $A$ and $B$ atoms constituting the rough interface [58]:

$$
V_{\sigma} \approx \mp \frac{1}{2}\left(m_{A}-m_{B}\right)-\frac{1}{2}\left(Z_{A}-Z_{B}\right)
$$

with the "+"-sign ("-"-sign) denoting the spin-up (spin-down) direction. This relationship enables one to put the above formulated requirement for a high GMR in quantitative terms i.e. band-structure calculations of the magnetic moments $m_{A}$ and $m_{B}$ at the rough interface may in principle be used to predict trends in the GMR with composition and growth orientation. So far this has not been done.

Although these are very promising developments, the relative importance of interface scattering with respect to bulk scattering remains a difficult issue which cannot be resolved alone by numerical calculations of the electronic structure or the electron scattering at rough interfaces. The relative contribution of the several scattering processes to the conductivity of a particular multilayer and related to this, their effect on the magnitude of the GMR ratio is determined, as we have mentioned already, by a complex interplay of several factors of which the properties of the intrinsic scattering processes is one. Many experiments performed in the last few years aimed at investigating and unrevealing the role of these other factors. Particularly, the problem of interface versus bulk scattering received much attention. Here, a number of approaches were followed to attack this problem from experimental point of view. We mention a few. (i) Studies in which the effect of the magnetic layer thickness is investigated. In the case that the scattering centers are distributed uniformly over the ferromagnet (predominant bulk scattering), the number of active scattering centers increases with the FM thickness (and thus the GMR ratio) as long as the thickness does not exceed the longest of the mean free path lengths. If it does, the inner part of the ferromagnetic layer becomes inactive and will contribute primarily to shunting. As a result a maximum in the MR ratio will occur at a relatively large thickness comparable to this mean free path. In contrast, for the case of predominant interface scattering, the GMR ratio is already large for FM thicknesses sufficient to exhibit the bulk electronic and magnetic properties. This thickness may be only a few monolayers. However, in practice the FM thickness dependencies for several material combinations which were investigated and analyzed using the Boltzmann transport model yielded inconclusive results: dependencies analyzed as supposedly being a result of pure interfacial scattering differed not spectacularly from analogous cases analyzed as pure bulk scattering [40]. (ii) To obtain information on the role of the interfaces, the roughness of the interfaces has been modified by giving the sam- 
ples anneal treatments or by depositing an alloyed layer at the interface. Although the experiments indicated that the degree of roughness is indeed important for the magnitude of the GMR ratio, again no conclusion could be drawn regarding the relative importance of bulk versus interface scattering. Furthermore, due to controversial experiments, it appeared difficult to determine a (general) relation between the roughness and the GMR. (iii) Another attempt to draw conclusions regarding the role of the interfaces involved the insertion of different elements at the interfaces. For example, Parkin started from $\mathrm{NiFe} / \mathrm{Cu}$ interfaces and studied the effect on the GMR of additions of small amounts of Co between the NiFe and the $\mathrm{Cu}$ layer. Addition of 3 monolayers appeared sufficient to increase the GMR by a factor of 2 [59]. Based on this it is tentative to draw the conclusion that interface scattering is the primary source for the GMR. However, what one in fact has shown with the experiment is that the spin-dependence of the scattering at a $\mathrm{Co} / \mathrm{Cu}$ interface is stronger than that for a $\mathrm{Ni}_{80} \mathrm{Fe}_{20} / \mathrm{Cu}$ interface. No conclusion can be drawn on the relative importance of bulk and interface scattering for the $\mathrm{Ni}_{80} \mathrm{Fe}_{20} / \mathrm{Cu}$ system.

A development which seems to offer a way out of this problem is that recently it has become possible to perform experiments in a different geometry viz. where the current flows perpendicular to the planes of the layers $[60,61]$ rather than the geometry considered so far in this paper where the current flows parallel to layer planes. For this geometry the theoretical analysis appears much easier: a model strongly resembling the two-current model proved sufficient to accurately describe the conductivity for this geometry [62]. As a consequence more accurate determinations of the respective contributions of the interface and bulk scattering to the GMR could be made. For an overview we refer to [63].

To conclude this article we mention that significant progress has been made in understanding the GMR phenomenon. However, several questions remain unanswered and require further research. Many of them are raised by the several theoretical approaches which are not discussed here. Issues that are unresolved include e.g. the role of quantum size effects, the effect of potential steps at the interfaces and the angular dependence of transmission coefficients.

\section{Acknowledgment}

The autors gratefully acknowledges the fellowship of the Royal Dutch Academy of Arts and Sciences. He also wishes to express his gratitude to the colleagues at Philips Research Laboratories and Eindhoven University of Technology for valuable discussions.

\section{References}

[1] W. Dürr, M. Taborelli, O. Paul, R. Germar, W. Gudat, D. Pescia, M. Landolt, Phys. Rev. Lett.62, 206 (1989); C.A. Ballentine, R.L. Fink, J. Araya-Pochet, J.L. Erskine, Phys. Rev. B 41, 2631 (1990); J.J. de Miguel, A. Cebollada, J.M. Gallego, S. Ferrer, R. Miranda, C.M. Schneider, P. Bressler, J. Garbe, K. Bethke, J. Kirschner, Surf. Sci. 211/212, 732 (1989).

[2] L. Néel, J. Phys. Rad. 15, 225 (1954). 
[3] See e.g. I.K. Schuller, in: Physics, Fabrication, and Applications of Multilayered Structures, Eds. P. Dhez, C. Weisbuch, Plenum Press, New York 1988, p. 139.

[4] B.Y. Jin, J.B. Ketterson, Adv. Phys. 38, 189 (1989).

[5] L.M. Falicov, D.T. Pierce, S.D. Bader, R. Goronsky, K.B. Hathaway, H.J. Hopster, D.N. Lambeth, S.S.P. Parkin, G. Prinz, M. Salamon, I.K. Schuller, R.H. Victora, J. Mater. Res. 59, 1299 (1990).

[6] Ultrathin Magnetic Structures, Eds. J.A.C. Bland, B. Heinrich, Springer, Berlin 1992.

[7] M. Rührig, R. Schäfer, A. Hubert, R. Mosler, J.A. Wolf, S. Democritov, P. Grünberg, Phys. Status Solidi A 125, 635 (1991); S. Democritov, J.A. Wolf, P. Grünberg, W. Zinn, Mater. Res. Soc. Proc. 231, 133 (1992).

[8] A. Yelon, in: Physics of Thin Films, Vol. 6, Academic, New York 1971, p. 205.

[9] M.B. Salamon, S. Sinha, J.J. Rhyne, J.E. Cunningham, R.W. Erwin, C.P. Flynn, Phys. Rev. Lett. 56, 259 (1986).

[10] C.F. Majkrzak, J.M. Cable, J. Kwo, M. Hong, D.B. Mcwhan, Y. Yafet, J.V. Waszczak, C. Vettier, Phys. Rev. Lett. 56, 2700 (1986).

[11] P. Grünberg, R. Schreiber, Y. Pang, M.B. Brodsky, H. Sowers, Phys. Rev. Lett. 57, 2442 (1986).

[12] P. Grünberg, J. Appl. Phys. 57, 3673 (1985).

[13] S.S.P. Parkin, N. More, K.P. Roche, Phys. Rev. Lett. 64, 2304 (1990).

[14] S.S.P. Parkin, D. Mauri, Phys. Rev. B 44, 7131 (1991).

[15] S.S.P. Parkin, Phys. Rev. Lett. 67, 3598 (1991).

[16] P.J.H. Bloemen, R. Coehoorn, W.J.M. de Jonge, J. Magn. Magn. Mater. 121, 306 (1993).

[17] M.T. Johnson, R. Coehoorn, J.J. de Vries, N.W.E. McGee, J. aan de Stegge, P.J.H. Bloemen, Phys. Rev. Lett. 69, 969 (1992).

[18] A. Schreyer, K. Bröhl, J.F. Ankner, Th. Zeidler, P. Bödeker, N. Metoki, C.F. Maikrzak, H. Zabel, Phys. Rev. B 47, 15334 (1993); M.A. Howson, B.J. Hickey, J. Xu, D. Grieg, N. Wiser, Phys. Rev. B 48, 1322 (1993).

[19] S.T. Purcell, W. Folkerts, M.T. Johnson, N.W.E. McGee, K. Jager, J. aan de Stegge, W.B. Zeper, W. Hoving, Phys. Rev. Lett. 67, 903 (1991).

[20] S.T. Purcell, M.T. Johnson, N.W.E. McGee, R. Coehoorn, W. Hoving, Phys. Rev. $B$ 45, 13064 (1992).

[21] M.T. Johnson, S.T. Purcell, N.W.E. McGee, R. Coehoorn, J. aan de Stegge, W. Hoving, Phys. Rev. Lett. 68, 2688 (1992); P.J.H. Bloemen, R. van Dalen, W.J.M. de Jonge, M.T. Johnson, J. aan de Stegge, J. Appl. Phys. 73, 5972 (1993).

[22] J. Unguris, R.J. Celotta, D.T. Pierce, J. Magn. Magn. Mater. 127, 205 (1993).

[23] A. Fuss, S. Democritov, P. Grünberg, W. Zinn, J. Magn. Magn. Mater. 103, L221 (1992).

[24] M. Ruderman, C. Kittel, Phys. Rev. 96, 99 (1954).

[25] T. Kasuya, Prog. Theor. Phys. 16, 45 (1956).

[26] K. Yosida, Phys. Rev. 106, 893 (1957).

[27] P. Bruno, J. Magn. Magn. Mater. 121, 248 (1993).

[28] R. Coehoorn, Phys. Rev. B 44, 9331 (1991). 
[29] C. Chappert, J.P. Renard, Europhys. Lett. 15, 553 (1991).

[30] D.M. Deaven, D.S. Rokhsar, M. Johnson, Phys. Rev. B 44, 5977 (1991).

[31] P. Bruno, J. Magn. Magn. Mater. 121, 248 (1993); P. Bruno, C. Chappert, Phys. Rev. Lett. 67, 1602 (1991); Phys. Rev. Lett. 67, 2592 (1991); P. Bruno, Phys. Rev. $B$ 46, 261 (1992).

[32] M.D. Stiles, Phys. Rev. B 48, 7238 (1993).

[33] S.N. Okuno, K. Inomata, Phys. Rev. Lett. 70, 1711 (1993); S.S.P. Parkin, C. Chappert, F. Herman, Europhys. Lett. 24, 71 (1993).

[34] P.J.H. Bloemen, M.T. Johnson, M.T.H. van de Vorst, R. Coehoorn, J.J. de Vries, R. Jungblut, J. aan de Stegge, A. Reinders, W.J.M. de Jonge, Phys. Rev. Lett. 72, 764 (1994).

[35] S.N. Okuno, K. Inomata, Phys. Rev. Lett. 72, 1553 (1994).

[36] J. Barnaś, J. Magn. Magn. Mater. 111, L215 (1992); J. Magn. Magn. Mater. 128, 171 (1994).

[37] P. Bruno, Europhys. Lett. 23, 615 (1993).

[38] J.J. de Vries, P.J.H. Bloemen, A.A.P. Schudelaro, R. Jungblut, A. Reinders, J. Kohlhepp, R. Coehoorn, W.J.M. de Jonge, Phys. Rev. Lett., accepted.

[39] M.T. Johnson, M.T.H. van de Vorst, P.J.H. Bloemen, R. Coehoorn, A. Reinders, J. aan de Stegge, R. Jungblut, Phys. Rev. Lett., accepted.

[40] A. Fert, P. Bruno, in: Ultrathin Magnetic Structures, Eds. J.A.C. Bland, B. Heinrich, Vol. 2, Springer, Berlin 1992, p. 82.

[41] S.S.P. Parkin, in: Ultrathin Magnetic Structures, Eds. J.A.C. Bland, B. Heinrich, Vol. 2, Springer, Berlin 1992, p. 148.

[42] B. Dieny, J. Magn. Magn. Mater. 136, 335 (1994).

[43] P.M. Levy, Solid State Physics, Eds. D. Turnbull, H. Ehrenreich, Vol. 47, Academic Press, San Diego 1995, p. 367.

[44] G. Binasch, P. Grünberg, F. Saurenbach, W. Zinn, Phys. Rev. B 39, 4828 (1989).

[45] M.N. Baibich, J.M. Broto, A. Fert, F. Nguyen Van Dau, F. Petroff, P. Eitenne, G. Creuzet, A. Friedrich, J. Chazelas, Phys. Rev. Lett. 61, 2472 (1988).

[46] D.H. Mosca, F. Petroff, A. Fert, P.A. Schroeder, W.P. Pratt, Jr., R. Laloee, S. Lequien, J. Magn. Magn. Mater. 94, L1 (1991).

[47] V.S. Speriosu, B. Dieny, P. Humbert, B.A. Gurney, H. Lefakis, Phys. Rev. B 44, 5358 (1991).

[48] R. Schad et al., Appl. Phys. Lett. 64, 3500 (1994).

[49] P. Chubing, Dai Chen, Dia Daosheng, J. Magn. Magn. Mater. 126, 400 (1993); M.L. Watson, W.D. Doyle, H. Fujiwara, J. Appl. Phys. 73, 5518 (1993).

[50] B. Rodmacq, A. Mallon, unpublished.

[51] I.A. Campbell, A. Fert, in: Ferromagnetic Materials, Ed. E.P. Wohlfarth, North-Holland, Amsterdam 1982.

[52] B. Dieny, V.S. Speriosu, S. Metin, S.S.P. Parkin, B. Gurney, P. Baumgart, D. Wilhoit, J. Appl. Phys. 69, 4774 (1991).

[53] J. Mathon, Contemp. Phys. 32, 143 (1991).

[54] N.F. Mott, Proc. R. Soc. London A 153, 699 (1936); 156, 368 (1936). 
[55] J.S. Dugdale, in: The Electrical Properties of Metals and Alloys, Edward Arnold, London 1977, p. 120.

[56] D.A. Papaconstantopoulos, Handbook of the Band Structure of Elemental Solids, Plenum, New York 1986.

[57] H. Itoh, J. Inoue, S. Maekawa, Phys. Rev. B 47, 5809 (1993); J. Inoue, S. Maekawa, Prog. Theor. Phys. Suppl. 106, 187 (1991); J. Inoue, H. Itoh, S. Maekawa, J. Phys. Soc. Jpn. 61, 1149 (1992); J. Inoue, A. Oguri, S. Maekawa, J. Magn. Magn. Mater. 104, 1883 (1992).

[58] R. Coehoorn, J. Magn. Magn. Mater. 121, 432 (1993); J. Magn. Magn. Mater., in press.

[59] S.S.P. Parkin, Phys. Rev. Lett. 71, 1641 (1993); Appl. Phys. Lett. 61, 1358 (1993).

[60] W.P. Pratt Jr., S.-F. Lee, J.M. Slaughter, R. Lololee, P.A. Schroeder, J. Bass, Phys. Rev. Lett. 66, 3060 (1991).

[61] M.A.M. Gijs, S.K.J. Lenczowski, J.B. Giesbers, Phys. Rev. Lett. 70, 3343 (1993).

[62] T. Valet, A. Fert, J. Magn. Magn. Mater. 121, 378 (1993).

[63] M.A.M. Gijs, in: Magnetic Thin Films and Industrial Applications, Springer Series in Materials Science, Ed. U. Hartman, Springer, Berlin 1996, to be published. 